\title{
Epidemiology of Rural/Urban Stroke Disparities in the Greater Cincinnati / Northern Kentucky Stroke Study
}

Adam Jasne MD, ${ }^{1}$ Cole Brokamp PhD, ${ }^{1,2}$ Kathleen Alwell BSN, ${ }^{1}$ Charles J. Moomaw PhD, ${ }^{1}$ Matthew L. Flaherty MD, ${ }^{1}$ Opeolu Adeoye MD MS, ${ }^{1}$ Daniel Woo MD MS, ${ }^{1}$ Jason Mackey MD MS, ${ }^{3}$ Simona Ferioli MD, ${ }^{1}$ Sharyl Martini MD PhD, ${ }^{4}$ Felipe de los Rios la Rosa MD, ${ }^{1,5}$ Brett M. Kissela MD MS, ${ }^{1}$ Jane Khoury PhD, ${ }^{1,2}$ Dawn Kleindorfer MD

1. University of Cincinnati, Cincinnati, $\mathrm{OH}$; 2. Cincinnati Children's Hospital Medical Center, Cincinnati, OH; 3. Indiana University, Indianapolis, IN; 4. Michael E. DeBakey VA Medical Center, Houston, TX; 5. Baptist Health Neuroscience Center, Miami, FL

\section{Introduction}

Stroke mortality is higher in rural areas than urban. Limited data exist as to why. We sought to evaluate whether the Greater Cincinnati/Northern Kentucky Stroke Study (GCNKSS) could be used to evaluate these disparities.

The GCNKSS is a population-based epidemiologic study capturing all ischemic stroke cases in region surrounding Cincinnati, Ohio, with a total population of 1.3 million. The study region includes Hamilton and Clermont counties in Ohio and Boone, Kenton, and Campbell counties in Kentucky. Census areas within the region contain a wide variety of population densities, but due to proximity to Cincinnati (in Hamilton County), few meet commonly-used rurality criteria.

\section{Methods}

- Identified all ischemic stroke cases among non-institutionalized individuals in the GCNK region in 2010

- Geocoded cases to census tract based on home address

- Evaluated association between census tractlevel stroke rate and tract characteristics:

- population density, rural \%, impervious development $\%$, socioeconomic deprivation index, age, race, and sex

- Analyzed association unadjusted and adjusted for age, race, and a composite socioeconomic deprivation index

\section{Figure 1. Region map, GCNKSS 2010}

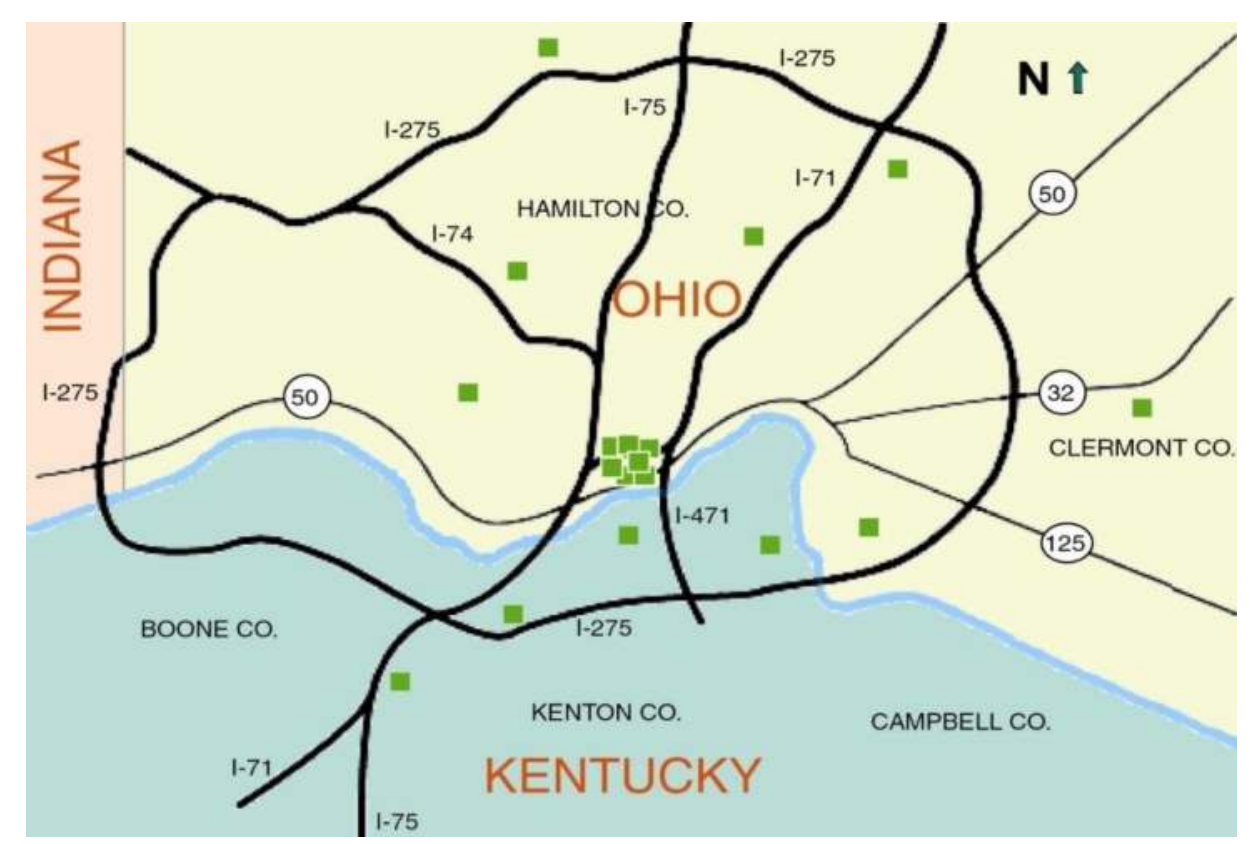

Geographic distribution of 15 hospitals in the Greater Cincinnati / Northern Kentucky Stroke Study region

\section{Results}

We identified 1,633 individuals who suffered a stroke during the study period

- Demographics: $54.1 \%$ female, $20.1 \%$ black

- County of residence: $63.1 \%$ Hamilton, $14.1 \%$ Clermont, $11.0 \%$ Kenton, 6.1\% Boone, 5.8\% Campbell

After adjustment for confounders, a quartile decrease in population density had a small but significant association with increased stroke rate.

A decrease of $\sim 17,000$ people/ $\mathrm{km}^{2}$ was associated with an increase of 1.5 strokes / 10,000 people / year.

\section{Figure 2. Unadjusted stroke rate} by census tract, GCNKSS 2010

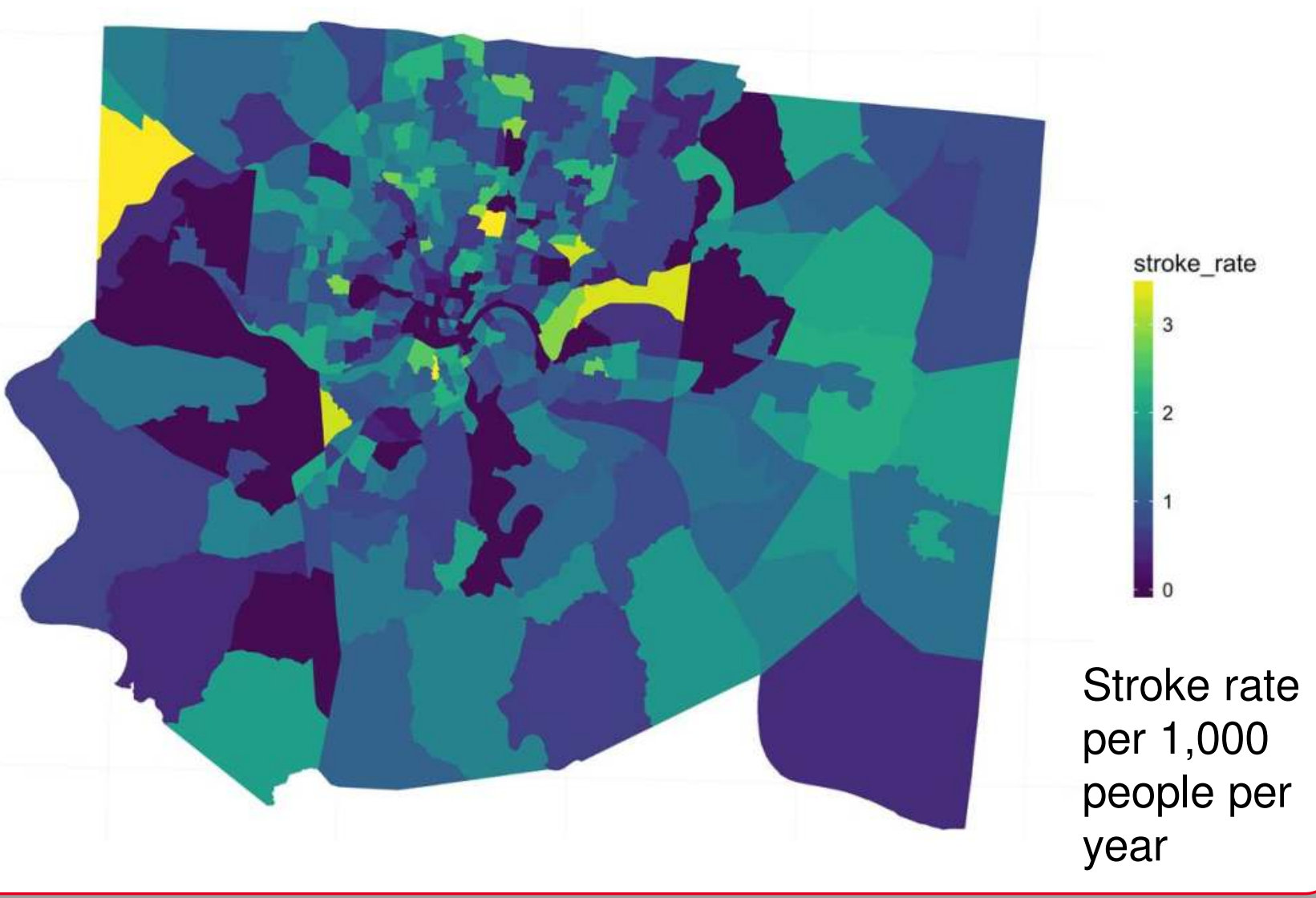

\section{Conclusions}

- Rural/urban stroke disparities persist even in areas such as GCNK, considered by some metrics to be non-rural.

- Our results support an understanding of rurality as a continuum, rather than a binary variable.

- Our results suggest that the GCNKSS can be used to study rural/urban stroke disparities.

- Future research should leverage the GCNKSS to evaluate factors that contribute to regional rural/urban disparities, how they relate to national and international disparities, and potential approaches to addressing them. 